\title{
A proposal for pathologic processing of breast implant capsules in patients with suspected breast implant anaplastic large cell lymphoma
}

\author{
Kirill A. Lyapichev $\mathbb{1}^{1} \cdot$ Sergio Piña-Oviedo ${ }^{2} \cdot$ L. Jeffrey Medeiros ${ }^{1} \cdot$ Mark G. Evans $\mathbb{1}^{3} \cdot$ Hui Liu ${ }^{4} \cdot$ Alonso R. Miranda $^{5} \cdot$ \\ Kelly K. Hunt ${ }^{6} \cdot$ Mark W. Clemens $^{7} \cdot$ John M. Stewart ${ }^{8} \cdot$ Mitual B. Amin $^{9} \cdot$ Andres E. Quesada $^{10} \cdot$ Siaw Ming Chai ${ }^{11}$. \\ Arianna Di Napoli $\mathbb{1}^{12} \cdot$ Arthy Yoga $^{13} \cdot$ Sanat K. Dave ${ }^{1} \cdot$ Ignacio I. Wistuba ${ }^{14} \cdot$ Yun Wu $^{8} \cdot$ Carlos E. Bueso-Ramos $^{1}$. \\ Ellen J. Schlette ${ }^{1} \cdot$ Maria C. Ferrufino-Schmidt ${ }^{14} \cdot$ Sanam Loghavi $\mathbb{D}^{1} \cdot$ Joseph D. Khoury $^{1}{ }^{1} \cdot$ Ken H. Young ${ }^{1}$. \\ Roberto N. Miranda (1) ${ }^{1}$
}

Received: 4 May 2019 / Revised: 28 June 2019 / Accepted: 29 June 2019 / Published online: 5 August 2019

(c) United States \& Canadian Academy of Pathology 2019

\begin{abstract}
Breast implant anaplastic large cell lymphoma is an entity recently recognized by the World Health Organization. The tumor arises around textured-surface breast implants and is usually confined to the surrounding fibrous capsule. Currently, there are no recommendations for handling and sampling of capsules from patients with suspected breast implant anaplastic large cell lymphoma without a grossly identifiable tumor. We analyzed complete capsulectomies without distinct gross lesions from patients with breast implant anaplastic large cell lymphoma. The gross appearance of the capsules as well as the presence, extent and depth of tumor cells on the luminal side and number of sections involved by lymphoma were determined by review of routine stains and CD30 immunohistochemistry. We then used a mathematical model that included the extent of tumor cells and number of positive sections to calculate the minimum number of sections required to identify $95 \%$ of randomly distributed lesions. We identified 50 patients with breast implant anaplastic large cell lymphoma who had complete capsulectomies. The implants were textured in all $32(100 \%)$ cases with available information. Anaplastic large cell lymphoma was found in 44/50 (88\%) capsules; no tumor was found in six (12\%) patients who had lymphoma cells only in the effusion. The median number of sections reviewed was 20 (range, 2-240), the median percentage of sections involved by tumor was 6\% (range, 0-90\%), and the median percentage of sections involved by lymphoma was 10\% (range, 0-90\%). Invasion deep into or through the capsule was identified in 18/50 (36\%) patients. In patients with breast implant anaplastic large cell lymphoma without a grossly identifiable tumor we identified a spectrum of involvement and we propose a protocol for handling, sampling and reporting these cases. The number of sections to exclude the presence of lymphoma with more than $95 \%$ certainty was supported by a mathematic rationale.
\end{abstract}

Breast implant anaplastic large cell lymphoma is a newly recognized, provisional entity in the 2017 revision of the World Health Organization classification of lymphoid tissues [1]. These tumors are of T-cell lineage, arise around textured-surface breast implants [2, 3] and clinically present as an effusion around the implant, or less frequently as a

Supplementary information The online version of this article (https:// doi.org/10.1038/s41379-019-0337-2) contains supplementary material, which is available to authorized users.

Roberto N. Miranda

Roberto.miranda@mdanderson.org

Extended author information available on the last page of the article mass or axillary lymphadenopathy [4]. Following the initial report in 1997 of breast implant anaplastic large cell lymphoma in association with silicone breast implants [5, 6], more than 600 cases are acknowledged by centralized agencies including the United States Food and Drug Administration [7]. The diagnosis should be suspected in patients with an effusion occurring $>1$ year after implant placement (delayed effusion, so-called "late seroma") and can be confirmed by cytologic examination of the effusion and/or by microscopic examination of the capsule [8]. However, there is evidence that the diagnosis of breast implant anaplastic large cell lymphoma can be missed by clinicians, oncologists, radiologists, and pathologists, and thus far there is no optimal screening method for diagnosis 
[9, 10]. Alleged reasons for underdiagnosis of breast implant anaplastic large cell lymphoma include the unusual features of this lymphoma, no previous history of lymphoma elsewhere and low awareness of this disease due to its recent recognition [9]. Another reason, from the pathology point of view is that capsule specimens often do not show grossly identifiable lesions and there are no guidelines to handle these specimens.

The last revised recommendations by the College of American Pathologists policy on "surgical specimens to be submitted to pathology for examination" (Appendix M), which include breast implants, were published in August 1995 when breast implant anaplastic large cell lymphoma was not yet defined [11]. In 1999, the College of American Pathologists surveyed 419 institutions and determined that $63 \%$ of specimens derived from "mammary implants" without mention of capsules were processed as "gross only", without requirement for microscopic analysis [12]. Furthermore, the recommendations for processing specimens by pathologists are not defined and appear to vary according to particular institutional bylaws. Recommendations in textbooks are for random sampling of two paraffin blocks when gross lesions are not identified, and of more blocks if lesions are identified [13]. Further sampling may be triggered by pathologists when neoplastic or atypical cells are unexpectedly found upon microscopic examination. Finally, Brody et al. [14] suggested in 2015 the need for a policy of College of American Pathologists regarding standardized pathologic processing of capsules associated with breast implants. Therefore, there is a need to update the College of American Pathologists recommendations for the pathologic management and handling of breast implants from patients with suspected breast implant anaplastic large cell lymphoma.

We believe that a major obstacle to developing recommendations for processing and handling capsulectomies of patients with breast implant anaplastic large cell lymphoma is the limited knowledge of the pathologic spectrum of the disease, that spans from cases with effusion only, to cases with superficial capsule involvement, to a grossly identifiable mass; less common is lymph node and rarely distant organ involvement. The handling of cases with a grossly identifiable mass may not pose a challenge in sampling. By contrast, handling cases without a grossly identifiable mass is more difficult. In this circumstance, there is uncertainty regarding what constitutes optimal sampling of the capsule surface and the number of sections required to properly diagnose or exclude disease. Since the extent or percentage of tumor involving fibrous capsules is unknown, one can predict that if the tumor is focal, random sampling may miss the tumor when insufficient sampling is performed. Alternatively, if the tumor occupies the entire surface of the luminal side of the capsule, a single section should be sufficient for diagnosis. Another dilemma occurs in cases with neoplastic cells found only in the effusion ("seroma") but not in the capsule as appears to occur in incipient cases, raising the concern for insufficient sampling of the specimen. The characteristic presentation of breast implant anaplastic large cell lymphoma as an effusion around an implant usually leads to the presumptive diagnosis of infection or ruptured implant by primary care physicians, radiologists, or plastic surgeons [10]. This opinion is furthered by the absence of a distinct mass or lymphadenopathy on physical examination or imaging studies, rendering a diagnosis of lymphoma as unlikely. Therefore, the microscopic diagnosis of lymphoma in most of these cases, presenting with an effusion and/or a mass, appears as an unexpected finding.

In this study, we analyzed a large series of breast capsule specimens from patients with breast implant anaplastic large cell lymphoma. We selected cases without grossly identifiable lesions, and reviewed the processing of each specimen. We then centrally reviewed the histologic sections of all cases to confirm the diagnosis, determined the extent and pathologic stage of disease and analyzed possible factors that may affect the optimal handling of these specimens. We also performed a mathematical analysis to estimate the minimum number of sections necessary to detect anaplastic large cell lymphoma on the surface of involved capsules without a grossly identifiable tumor mass. Based on these results, we propose a method for handling and sampling of these specimens and we suggest a checklist for reporting cases of breast implant anaplastic large cell lymphoma.

\section{Materials and methods}

This is a multi-institutional study where investigators contributed cases of breast implant anaplastic large cell lymphoma and provided gross pathologic information and all histologic sections used at time of diagnosis; these materials were centrally reviewed. The minimal histological criteria to diagnose breast implant anaplastic large cell lymphoma includes the presence of individual, clusters or sheets of large, pleomorphic cells with oval to lobated nuclei with vesicular chromatin, usually with prominent nucleoli and a moderate amount of clear or light blue cytoplasm; these tumor cells are uniformly found on the luminal surface of the capsule. Some cases show these cells into or throughout the capsule as invasive clusters. CD30 immunohistochemistry highlights the membrane and cytoplasm with a frequent Golgi pattern of all cells. Necrosis is detected in all cases, and it appears as granular material or as ghost cells that are strongly positive for CD30. Furthermore, clinically, most patients present with effusion "seroma" around breast implants. In patients with a tumor mass beyond the capsule, 
we required in addition identification of tumor cells at the luminal side of the capsule. All cases have been published $[4,8,15]$. The collected information included clinical presentation and type of implant (smooth vs textured surface; silicone vs saline filling). Only cases with complete capsulectomy were included. We excluded cases with partial capsulectomy or where a gross lesion or mass was identified. The total number of sections of capsule submitted per specimen was extracted. When possible, we measured the average length of sections of capsules. Occasional extreme variability was noted in the number of sections because in some cases resampling occurred, and the number of blocks and sections increased significantly. Histologic sections were assessed for extent of luminal surface available for evaluation, presence and extent of lymphoma cells, and relationship with deep margins. We reviewed all microscopic sections of each case included in this series and assessed the quality of the sections depending on the percentage of sections exhibiting the luminal surface of the capsule. We considered sampling optimal if an average of $\geq 50 \%$ of each capsule section showed the luminal surface; adequate if $10-49 \%$ showed the luminal side, and inadequate if $0-9 \%$ of the section displayed the luminal surface. We assessed the percentage of lymphoma cells on the luminal side of the capsule using histopathologic features and CD30 immunohistochemistry. We also documented the presence or absence of tumor cells in each section, and when positive, we recorded the percentage of capsule involved by lymphoma cells.

The percentage of evaluable luminal surface from the total length of the tissue section was deemed essential since it is apparent that this lymphoma is localized initially to the luminal surface of capsule and eventually infiltrates into and through the capsule with progression of disease. Similarly, the presence and extent of lymphoma cells relative to the evaluable luminal surface was assessed. The pathologic stage was determined following modified criteria proposed by Clemens et al. $[9,15]$. We assigned a T0 stage for those cases with cytology only positive, while the capsule was negative for lymphoma cells; cases in this category were previously lumped with cases with tumor cells lining on the surface of capsules (stage T1).

Immunohistochemical analysis for CD30 (Ber-H2, Agilent Technologies, Santa Clara, California, USA) was performed using fixed, paraffin embedded tissue, heatinduced epitope antigen retrieval, an avidin-biotin complex method, and an automated immunostainer (Leica Biosystems, Buffalo Grove, Illinois, USA).

The control group consisted of 10 patients who had breast implants and presented with effusion around the implant $n=$ $10)$, more than 1 year after implant placement. We selected cases who had complete capsulectomy, with or without cytologic or flow cytometry immunophenotypic analysis, and collected clinical data as indicated for patients who had anaplastic large cell lymphoma. The final pathologic diagnosis for all these cases was negative for lymphoma.

The Institutional Review Board at MD Anderson Cancer Center approved this study.

\section{Mathematical model}

Since identification of lymphoma cannot be determined by simple gross evaluation, there is a need to sample randomly for histological examination. The first question to address is what is a reasonable number of sections to take? We could then consider which areas are more likely to yield tumor, allowing for preferential sampling. A simulation with a regression was used to model the situation [16, 17].

In a regression model, there are two constants b0 and b1 that minimize the error between a line and all the data points where $b l$ is the y-intercept and $b 0$ is the slope. In this particular case we did not perform a linear regression on the raw data, but we did complete a regression on 1/coverage $\%$ because the data looked more appropriately represented with a $1 / x$ style of graph. The resultant curve that best fits the data was: $y=\mathrm{b} 0 / x+\mathrm{b} 1$

For the y data points, we used the regular $y$-values which in this case were the $95 \%$ percentile for the number of samples until we found the detection region. For the $x$-values, we used the inverse of the coverage $\%$, which explains why coverage $\%$ is shown in the denominator. For this reason the value of $b 0$ has the unusual real world interpretation as the increase in the number of samples needed as the reciprocal of the coverage $\%$ increases by $1 . b 1$ (horizontal asymptote) could be interpreted as the absolute minimum number of samples required even as the coverage $\%$ reaches 100 . The values were determined from a regression between the $95 \%$ quartile for the number of samples and the inverse of the coverage $\%$.

\section{Results}

A total of 50 patients with breast implant anaplastic large cell lymphoma who underwent total capsulectomy without a grossly identifiable mass were identified between January 1997 and December 2017. All cases were reviewed in the Department of Hematopathology of MD Anderson Cancer Center.

A summary of the relevant clinical information for these patients is shown in Table $1[8,9,15]$. The median age of patients was 52.5 years (range, 35-77 years). The reason for the implant was provided in 48 cases and was cosmetic in $27(56 \%)$ and reconstructive in $21(44 \%)$. The implant surface information was provided in 32 patients and all were textured $(100 \%)$. The filling of the implant was provided in 45 cases: silicone in $24(53 \%)$ and saline in 21 
Table 1 Clinical and pathologic findings of 50 patients with breast implant anaplastic large cell lymphoma

\begin{tabular}{|c|c|c|}
\hline Features & $n$ & $\%$ \\
\hline \multicolumn{3}{|l|}{ Clinical presentation $(n=50)$} \\
\hline Effusion & 50 & 100 \\
\hline Mass & 0 & 0 \\
\hline \multicolumn{3}{|l|}{ Side of lymphoma $(n=50)$} \\
\hline Left & 23 & 46 \\
\hline Right & 26 & 52 \\
\hline Bilateral & 1 & 2 \\
\hline \multicolumn{3}{|l|}{ Reason for implant $(n=48)$} \\
\hline Cosmetic & 27 & 56 \\
\hline Reconstruction & 21 & 44 \\
\hline \multicolumn{3}{|l|}{ Surface of implants $(n=32)$} \\
\hline Textured & 32 & 100 \\
\hline Smooth & 0 & 0 \\
\hline \multicolumn{3}{|l|}{ Filling of implant $(n=45)$} \\
\hline Silicone & 24 & 53 \\
\hline Saline & 21 & 47 \\
\hline Ruptured implant $(n=31)$ & 2 & 6 \\
\hline \multicolumn{3}{|l|}{ Pathologic stage $^{\mathrm{a}}(n=50)$} \\
\hline $\mathrm{T} 0$ & 6 & 12 \\
\hline $\mathrm{T} 1$ & 13 & 26 \\
\hline $\mathrm{T} 2$ & 13 & 26 \\
\hline $\mathrm{T} 3$ & 13 & 26 \\
\hline \multirow[t]{2}{*}{$\mathrm{T} 4$} & 5 & 10 \\
\hline & $\begin{array}{l}\text { Median size of capsule, } \\
\text { maximum diameter }(\mathrm{cm})\end{array}$ & Range $(\mathrm{cm})$ \\
\hline \multirow{2}{*}{$\begin{array}{l}\text { Size of capsules } \\
(n=44)\end{array}$} & 13 & $3.0-18.0$ \\
\hline & Median section size $(\mathrm{cm})$ & Range $(\mathrm{cm})$ \\
\hline $\begin{array}{l}\text { Size of sections } \\
(n=50)\end{array}$ & 2.1 & $1.0-3.0$ \\
\hline
\end{tabular}

${ }^{\text {a Pathologic stage according to Clemens et al. [15] and Quesada et al. [9] }}$ Pathologic stage T0 was created to include cases with effusion only, without lymphoma cells in capsules

(47\%). All patients presented with effusion and no patients had a grossly identifiable mass.

The gross description in most cases was limited to the size of the specimen and is displayed in Table 1. The recorded size of the capsules was available in 44/50 (88\%) cases. The median largest diameter of the capsules was 13.0 $\mathrm{cm}$ (range, $3.0-18.0 \mathrm{~cm} ; n=44$ cases), and the median size of the second largest diameter of the capsules was $11.0 \mathrm{~cm}$ (range, $2.5-15.5 \mathrm{~cm} ; n=39$ cases). A summary of relevant gross and histological information of 50 patients is shown in Table 2. The median number of paraffin blocks was 8 (range, 2-147), and the median number of sections of capsule was 20 (range, 2-240). The median length of capsule sections was $2.1 \mathrm{~cm}$ (range, $1.0-3.0 \mathrm{~cm}, n=50$ cases evaluated). Tumor cells were detected in $44 / 50$ (88\%) capsules; the remaining six $(12 \%)$ cases had lymphoma cells only in the effusion and the capsules did not have lymphoma cells. The median number of sections submitted for these cases was 24 (range, 12-51 sections). The median number of sections with identifiable tumor was 6 (range, 1-90) and the median percentage of the luminal surface involved by lymphoma was $10 \%$ (range, $0-90 \%$ ). The quality of sections as determined by the orientation of sections displaying the luminal side of capsules was excellent in $16(32 \%)$, adequate in $30(62 \%)$, and poor in four $(8 \%)$ cases. Of interest, in $2 / 4(50 \%)$ cases with poorly oriented capsules no lymphoma cells were identified. For microscopically identifiable tumor in capsules, it was not possible to map the location of the tumor and, if there was more than one positive section, it was not possible to determine if the affected sections were contiguous or skipped. It is apparent that the number of sections does not necessarily reflect the initial number of sections taken, since in certain cases, additional sampling and larger number of sections were prompted after a finding of lymphoma either in cytologic specimens or in microscopic foci of lymphoma in capsules. The absence of tumor involvement in capsules in some cases may have resulted from suboptimal orientation of the sections.

Pathologic tumor stage revealed no tumor in the capsule (T0) in six (12\%) and the diagnosis was established because of positive cytology (Fig. 1a). Tumor was present in the superficial layer of the luminal side (T1) of the capsule in 13 (26\%), tumor breaching the superficial layer (T2) in 13 (26\%), tumor deeply invading into the capsule (T3) in 13 (26\%), and tumor beyond capsule (T4) in five (10\%) cases. (Table 1 and Fig. 1b-f) For the six cases with tumor identified only in the cytology specimen, although no tumor cells were identified on the luminal surface of the capsule, granular pink material was focally attached to the lumen that was CD30-positive by immunohistochemistry (Fig. 1b).

The control group consisted of 10 patients for whom the clinical and pathologic features are summarized in Table 3. All patients presented with effusion more than 1 year after implant placement, and all with available information had textured implants $(n=8)$. All patients had complete capsulectomy. The luminal side of the capsules revealed synovium-like lining (also referred to as synovial metaplasia) (Fig. 2a left) in six patients, while no synovium-like cells were noted in four patients (Fig. 2a right). No large pleomorphic cells or necrosis was observed. Inflammatory cells including small lymphocytes, histiocytes and plasma cells in the stroma of capsule was noted in nine patients. Immunohistochemistry for CD30 revealed that six cases did not have any positive cells. Four cases had a 1-6 (median, 1) CD30-positive cells per 10 high power fields, all small 
Table 2 Extent and pathologic stage of lymphoma in complete capsulectomies from patients without grossly identifiable breast implant anaplastic large cell lymphoma

\begin{tabular}{|c|c|c|c|c|c|c|c|c|}
\hline Case \# & $\begin{array}{l}\text { Quality of } \\
\text { luminal surface }^{a}\end{array}$ & $\begin{array}{l}\text { Presence of } \\
\text { lymphoma cells in } \\
\text { capsule }^{b}\end{array}$ & $\begin{array}{l}\% \text { of capsule } \\
\text { involved by } \\
\text { lymphoma }\end{array}$ & $\begin{array}{l}\text { Number of } \\
\text { sections } \\
\text { examined }\end{array}$ & $\begin{array}{l}\text { Number of sections } \\
(+) \text { for lymphoma }\end{array}$ & $\begin{array}{l}\% \text { sections }(+) \text { for } \\
\text { lymphoma }\end{array}$ & $\begin{array}{l}\text { Average size of } \\
\text { sections }(\mathrm{cm})\end{array}$ & $\begin{array}{l}\text { Pathologic stage } \\
(\mathrm{T} 0-\mathrm{T} 4)^{\mathrm{c}}\end{array}$ \\
\hline 1 & Excellent & No & 0 & 15 & 0 & 0 & 1.3 & 0 \\
\hline 2 & Adequate & No & 0 & 51 & 0 & 0 & 1 & 0 \\
\hline 3 & Excellent & No & 0 & 42 & 0 & 0 & 1.4 & 0 \\
\hline 4 & Excellent & No & 0 & 24 & 0 & 0 & 1.4 & 0 \\
\hline 5 & Poor & No & 0 & 24 & 0 & 0 & 1.5 & 0 \\
\hline 6 & Poor & No & 0 & 12 & 0 & 0 & 1.5 & 0 \\
\hline 7 & Adequate & Yes & 5 & 12 & 7 & 58 & 2.1 & 1 \\
\hline 8 & Excellent & Yes & 70 & 12 & 4 & 33 & 2 & 1 \\
\hline 9 & Adequate & Yes & 30 & 7 & 3 & 43 & 2 & 1 \\
\hline 10 & Adequate & Yes & 5 & 32 & 3 & 9 & 2 & 1 \\
\hline 11 & Adequate & Yes & 30 & 93 & 60 & 64 & 3 & 1 \\
\hline 12 & Adequate & Yes & 5 & 63 & 10 & 16 & 2.4 & 1 \\
\hline 13 & Adequate & Yes & 5 & 12 & 1 & 8 & 1.7 & 1 \\
\hline 14 & Adequate & Yes & 10 & 7 & 3 & 43 & 2 & 1 \\
\hline 15 & Excellent & Yes & 20 & 4 & 2 & 50 & 2 & 1 \\
\hline 16 & Adequate & Yes & 5 & 14 & 1 & 7 & 1.6 & 1 \\
\hline 17 & Adequate & Yes & 1 & 102 & 20 & 20 & 2.5 & 1 \\
\hline 18 & Excellent & Yes & 1 & 28 & 1 & 4 & 1.7 & 1 \\
\hline 19 & Excellent & Yes & 10 & 51 & 2 & 4 & 2 & 1 \\
\hline 20 & Excellent & Yes & 80 & 2 & 2 & 100 & 2 & 2 \\
\hline 21 & Adequate & Yes & 10 & 50 & 10 & 20 & 2.5 & 2 \\
\hline 22 & Adequate & Yes & 50 & 20 & 10 & 50 & 2.5 & 2 \\
\hline 23 & Adequate & Yes & 20 & 10 & 7 & 70 & 2.1 & 2 \\
\hline 24 & Adequate & Yes & 10 & 51 & 51 & 100 & 3 & 2 \\
\hline 25 & Adequate & Yes & 50 & 2 & 1 & 50 & 1.8 & 2 \\
\hline 26 & Excellent & Yes & 10 & 52 & 52 & 100 & 3 & 2 \\
\hline 27 & Excellent & Yes & 50 & 60 & 60 & 100 & 3 & 2 \\
\hline 28 & Adequate & Yes & 10 & 40 & 20 & 50 & 2.6 & 2 \\
\hline 29 & Adequate & Yes & 80 & 10 & 10 & 100 & 2.5 & 2 \\
\hline 30 & Adequate & Yes & 5 & 33 & 2 & 6 & 2 & 2 \\
\hline 31 & Excellent & Yes & 70 & 10 & 10 & 100 & 2.5 & 2 \\
\hline 32 & Adequate & Yes & 10 & 10 & 5 & 50 & 2 & 2 \\
\hline 33 & Poor & Yes & 40 & 3 & 2 & 67 & 2 & 3 \\
\hline 34 & Excellent & Yes & 10 & 12 & 1 & 8 & 2 & 3 \\
\hline 35 & Adequate & Yes & 70 & 10 & 10 & 100 & 2.5 & 3 \\
\hline 36 & Excellent & Yes & 50 & 27 & 27 & 100 & 3 & 3 \\
\hline 37 & Adequate & Yes & 70 & 19 & 14 & 74 & 2.5 & 3 \\
\hline 38 & Poor & Yes & 70 & 22 & 17 & 77 & 2.5 & 3 \\
\hline 39 & Excellent & Yes & 80 & 10 & 9 & 90 & 2.4 & 3 \\
\hline 40 & Adequate & Yes & 20 & 28 & 5 & 18 & 2 & 3 \\
\hline 41 & Adequate & Yes & 80 & 38 & 30 & 79 & 3 & 3 \\
\hline 42 & Adequate & Yes & 80 & 11 & 9 & 82 & 2.4 & 3 \\
\hline 43 & Adequate & Yes & 50 & 130 & 90 & 69 & 3 & 3 \\
\hline 44 & Adequate & Yes & 20 & 66 & 60 & 91 & 3 & 3 \\
\hline 45 & Excellent & Yes & 90 & 20 & 14 & 70 & 2.5 & 3 \\
\hline 46 & Adequate & Yes & 30 & 18 & 5 & 28 & 2 & 4 \\
\hline 47 & Adequate & Yes & 30 & 6 & 2 & 33 & 2 & 4 \\
\hline 48 & Excellent & Yes & 30 & 240 & 5 & 2 & 2.1 & 4 \\
\hline 49 & Adequate & Yes & 60 & 16 & 10 & 63 & 2.5 & 4 \\
\hline \multirow[t]{2}{*}{50} & Adequate & Yes & 5 & 26 & 8 & 31 & 2.3 & 4 \\
\hline & & $(+): 44(88 \%)$ & $\begin{array}{l}\text { Median } 10 \% ; \\
\text { Range: } 0-90 \%\end{array}$ & $\begin{array}{l}\text { Median, } 20 ; \\
\text { Range: } 2-240\end{array}$ & $\begin{array}{l}\text { Median, 6; } \\
\text { Range: 0-90 }\end{array}$ & $\begin{array}{l}\text { Median } 46.5 \% \\
\text { Range } 0-100 \%\end{array}$ & $\begin{array}{l}\text { Median section size } 2.1 \\
\mathrm{~cm} \text {; Range } 1.0-3.0 \mathrm{~cm}\end{array}$ & $\begin{array}{l}36 \% \text { invasive cases } \\
\text { (pathologic stage } \\
3 \text { and } 4 \text { ) }\end{array}$ \\
\hline
\end{tabular}

${ }^{\mathrm{a}}$ Assessment of the quality of orientation of capsules of patients with breast implant ALCL with total capsulectomy: Excellent: $\geq 50 \%$ of sections display luminal surface; Adequate: $10-49 \%$ of sections show luminal surface; Poor: $<10 \%$ of capsule sections exhibit luminal surface

${ }^{b}$ Negative cases include capsule without identifiable lymphoma cells; lymphoma cells were only in the cytology specimen

${ }^{c}$ Pathologic stage according to Clemens et al. [15], and Quesada et al. [9]. Pathologic stage T0 modified to include cases with effusion only, without lymphoma cells in capsules 
a

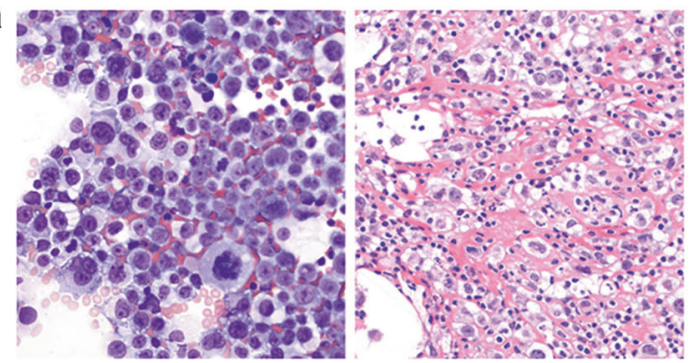

b

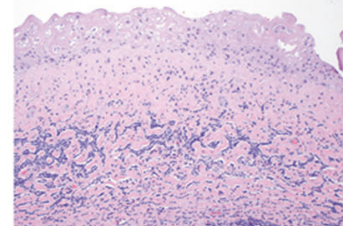

C

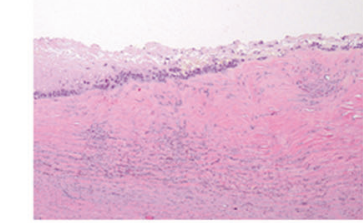

d

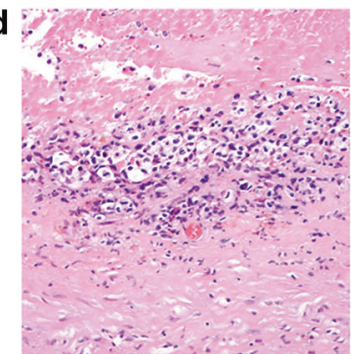

e

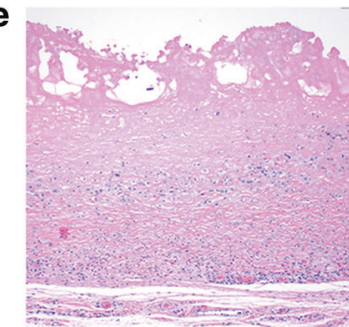
and

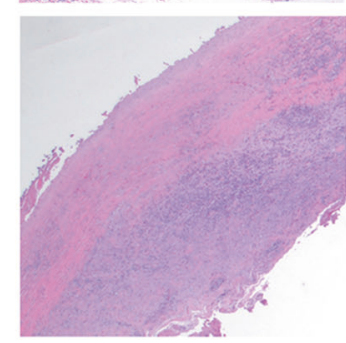

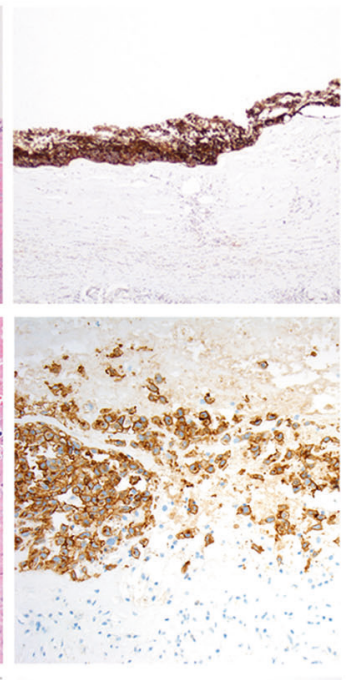
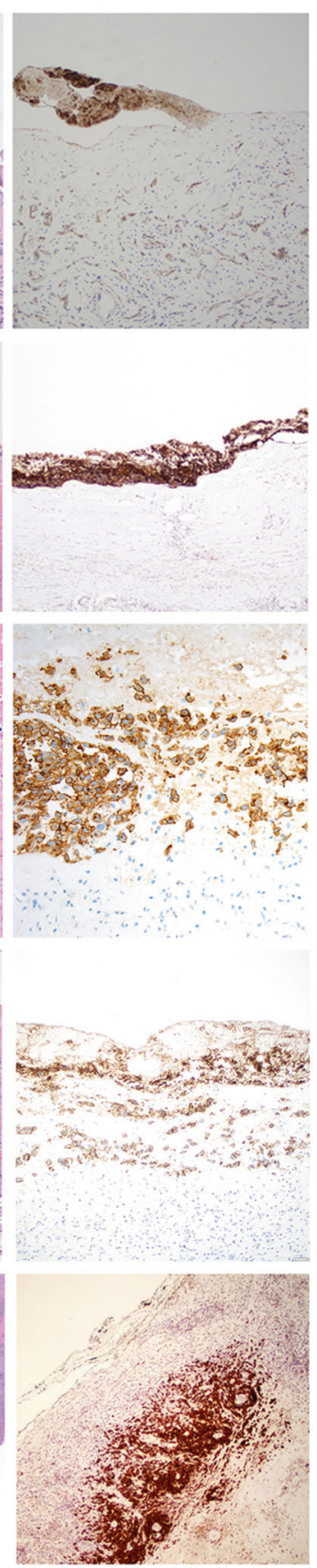

lymphocytes or plasma cells; no large pleomorphic cells were found (Fig. 2b left). Cytologic examination from 9 patients was negative and showed small lymphocytes

Fig. 1 Pathologic staging of capsulectomies in patients with breast implant anaplastic large cell lymphoma. a Left: Cytologic smear of effusion displays large pleomorphic cells with abundant basophilic cytoplasm containing vacuoles. Wright Giemsa stain, $\times 500$. Right: Cell block of effusion displays large pleomorphic cells with a fibrinoid background. Hematoxylin and eosin stain, $\times 400$. b Luminal side of capsule devoid of lymphoma cells (stage T0); the diagnosis in this case was based on positive cytology in the effusion. (Left: Hematoxylin and eosin, $\times 200$; Right: CD30 immunohistochemistry, $\times 200$ ). c Representative section of capsule involved by breast implant anaplastic large cell lymphoma displays the entire luminal surface has anaplastic large cell lymphoma cells, consistent with excellent orientation and proper "on edge" embedding (stage T1) (left). The neoplastic cells are admixed with abundant granular and necrotic material representing karyorrhectic tumor, further confirmed with CD30 immunohistochemistry (Left. hematoxylin and eosin, $\times 200$; Right. CD30 immunohistochemistry with hematoxylin counterstain, $\times 200$ ). d The lymphoma cells involve the luminal side of the capsule (stage T2) in which tumor cells infiltrate superficially into the capsule. (Left: hematoxylin and eosin, $\times 400$; Right: CD30 immunohistochemistry, $\times 400$ ). e The lymphoma cells infiltrate deep into the capsule (stage T3) in which lymphoma cells are admixed with reactive inflammatory cells. (Left: hematoxylin and eosin, $\times 200$; Right: CD30 immunohistochemistry, $\times 200)$. f In this low magnification, the lymphoma cells extend beyond the capsule (T4) in which tumor cells form large clusters throughout the capsule. (Left: hematoxylin and eosin, $\times 40$; Right: CD30 immunohistochemistry, $\times 40$ )

Table 3 Clinical and pathologic features of 10 patients with breast implants negative for lymphoma

\begin{tabular}{lll}
\hline Features & $n$ & $\%$ \\
\hline Clinical Presentation $(n=10)$ & & 100 \\
$\quad$ Effusion & 10 & \\
Side of implant $(n=10)$ & & 0 \\
$\quad$ Left & 0 & 10 \\
$\quad$ Right & 1 & 90 \\
$\quad$ Bilateral & 9 & 57 \\
Reason for implant $(n=7)$ & & 43 \\
$\quad$ Cosmetic & 4 & \\
$\quad$ Reconstruction & 3 & 100 \\
Surface of implants $(n=7)$ & & 0 \\
$\quad$ Textured & 7 & \\
$\quad$ Smooth & 0 & 100 \\
Filling of implant $(n=9)$ & & 0 \\
$\quad$ Silicone & 9 & \\
$\quad$ Saline & 0 & 60 \\
Histologic features & & 40 \\
Surface lining $(n=10)$ & & \\
$\quad$ Synovium like & & \\
Fibrotic & & \\
Inflammation in capsule & & \\
$\quad$ Yes & & \\
$\quad$ No & & \\
\hline
\end{tabular}

admixed with histiocytes and occasional neutrophils. (Fig. 2b right) Flow cytometry immunophenotype was negative for aberrant $\mathrm{T}$ cells in six patients tested. 


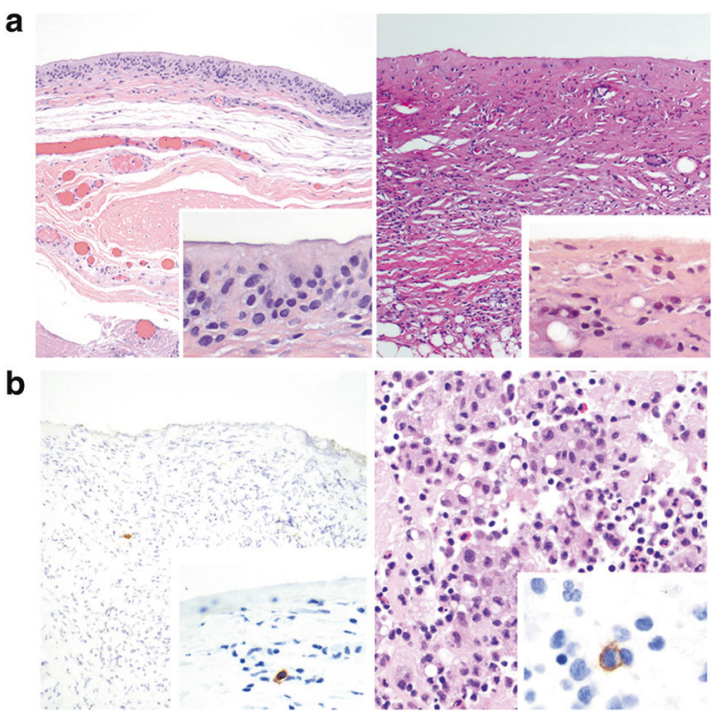

Fig. 2 a Histopathology of capsules from patients who presented with peri-implant effusion, negative for anaplastic large cell lymphoma. Left: Section of capsule lined by synovium-like cells (also referred to as synovial metaplasia). Inset shows detail of synovium-like cells with a cylindrical to cuboidal shape, indistinct cytoplasm, and round to oval nuclei of the luminal side of the capsule. The underlying stroma is loose and lacks inflammatory cells. (Hematoxylin and eosin, $\times 100$; inset $\times 400$ ). Right: Section of capsule devoid of synovium-like cells, but displays underlying fibrosis, chronic inflammation and small round clear spaces related with silicone material. Inset shows high magnification of the luminal surface, with a thin fibrous layer, almost acellular. (Hematoxylin and eosin, $\times 100$; inset $\times 400$ ). b Left: Immunohistochemistry for CD30 in a capsule negative for anaplastic large cell lymphoma highlights a single cell in the stroma. No cells are highlighted in the luminal side of the capsule. Inset: A single small lymphocyte is highlighted in the stroma. (Immunohistochemistry for CD30, $\times 100$; inset, immunohistochemistry for CD30, $\times 400$ ). Right: Cell block of peri-implant effusion shows small lymphocytes, histiocytes and neutrophils. Inset: Immunohistochemistry for CD30 in cell block highlights a single small to intermediate size cell among other inflammatory cells. (Hematoxylin and eosin, $\times 400$; CD30 immunohistochemistry, $\times 1000$ )

\section{Mathematical model}

The volume of implants varies according to patient breast size and patient preferences or anatomic restrictions; however a common range is between 200 and $400 \mathrm{~mL}$. Once introduced, the implant will be surrounded by a fibrous capsule that is stretched around the implant when removed en bloc. In our experience, the major diameters of capsules from en bloc specimens reflect the larger dimensions of the underlying implant $(14 \times 12 \mathrm{~cm})$. Freshly opened capsules shrink immediately and had an average of $17 \times 15 \mathrm{~cm}$ that may shrink further after fixation. However, review of pathologic reports does not usually specify the timing of measuring the major gross dimensions of capsules. Further variation may depend on intraoperative features, the presence of effusion or tumor, or implant preservation.
Fragmented capsules were not unusual, and for this type of specimen the word of the surgeon is followed to consider the specimen a complete capsulectomy.

In a simulation of a $14 \times 12 \mathrm{~cm}$ capsule with a grossly unidentifiable breast implant anaplastic large cell lymphoma, a $2.0 \times 2.0 \mathrm{~cm}$ square "tumor" is displayed on a flat capsule that corresponds to $\sim 2 \%$ of the capsule surface. In order to simulate the sampling procedure, we randomly placed a $2.0 \times 0.2 \mathrm{~cm}$ rectangle representing an average section size. Overlapping of the "sample rectangle" with the "tumor square" may occur randomly at various frequencies depending on the size of the "tumor" as shown in Fig. 3a. On a continuum of random "sampling", the "tumor" may be hit/detected and a number (of sections) will be recorded when the first hit occurs. In this model, the process is repeated 10,000 times and then the 95th percentile of that data is recorded. Finally, a scatter plot is generated between the 95th percentile of the number of samples required until first detection and the "percentage of capsular involvement by tumor". The plot suggests a reasonable number of samples to take in order to be confident of the presence/absence of tumor in the breast implant capsule.

The curve shows that a larger area of involvement by lymphoma correlates with a decreased number of events to hit/detect a lesion. Figure $3 b$ shows that the distribution of first detection strongly resembles a geometric distribution. For example, if the capsule has about $30 \%$ involvement (which would be a region of approximately $7 \mathrm{~cm} \times 7 \mathrm{~cm}$ ) then we can input that value into the formula and generate a result of 7.15 samples required, a number that can be rounded to 7 (Table 4). Therefore, if seven samples are taken, we can be $95 \%$ certain that if we have not found lymphoma by then, odds are we would not find it by continuing to take more samples. This simulation approach lets us confidently exclude capsule involvement by a very small percentage of tumor which could be missed if an inappropriately small number of sections is taken and also help to decrease the cost associated with an undefined number of sections for histologic processing that may occur with oversampling.

The resultant scatter plot closely resembled an inverse function equation so we performed a simple regression and found a suitable equation. We describe a simplified model of how to place reasonable upper limits and how many sections are needed before finding a positive one (i.e. lymphoma); we also used only 1 decimal in the formula:

$$
\text { Samples }=3.6+106.8 /(\text { Coverage } \%)
$$

An example on how to use this formula and details of some other cases with different areas of involvement by lymphoma and the calculated number of sections that randomly may detect such areas is displayed in Table 4. 


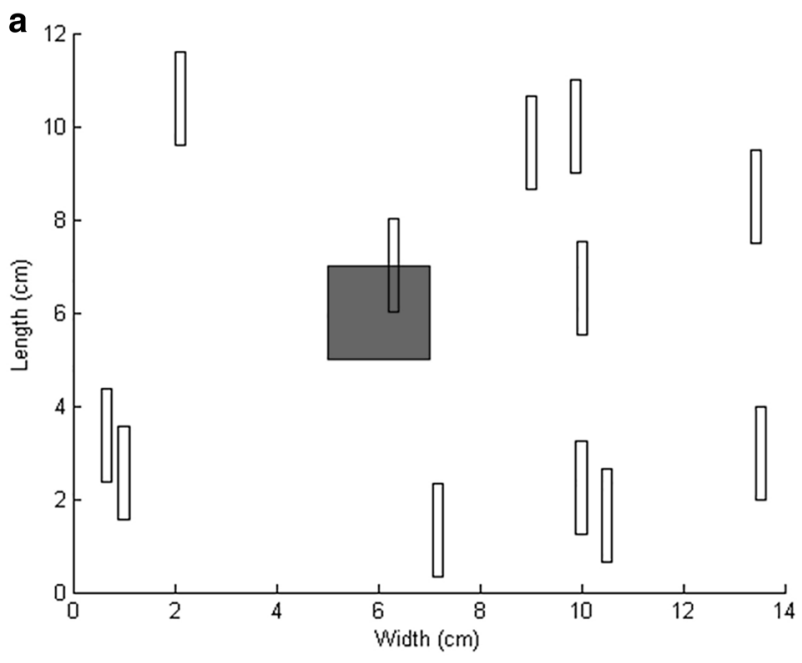

b

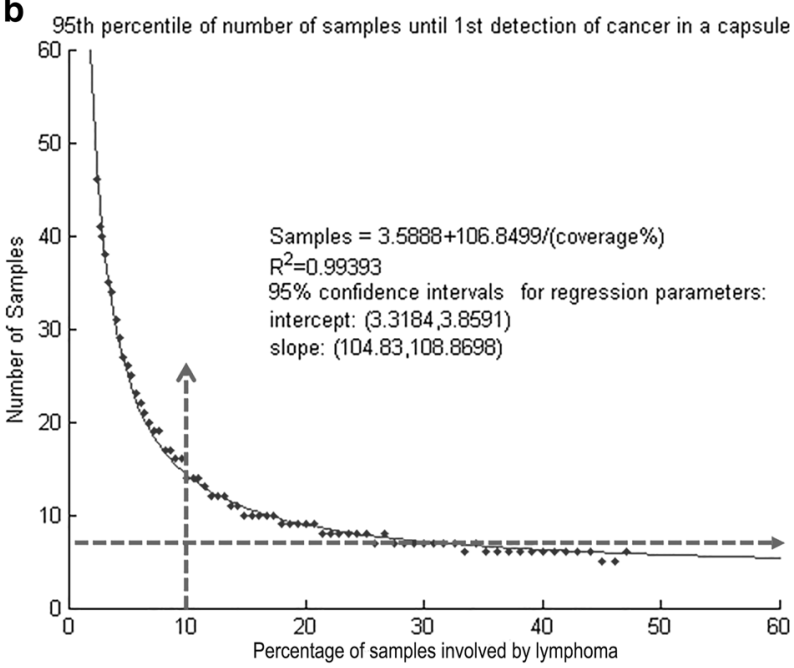

Fig. 3 a Mathematical approach to calculate the minimum number of sections (samples) to identify lymphoma cells in grossly unidentifiable cases of breast implant anaplastic large cell lymphoma. The grey square represents an hypothetical area of involvement by anaplastic large cell lymphoma, and the multiple white rectangles represent random sections to be taken in order to identify microscopic presence of anaplastic large cell lymphoma. Notice that only one white rectangle overlaps with the grey square. In the mathematical model, the sampling stopped as soon as one white rectangle overlapped/hit the grey square (area involved by anaplastic large cell lymphoma). b Mathematical approach to calculate the minimal number of sections to identify lymphoma cells in grossly unidentifiable cases of breast implant anaplastic large cell lymphoma. These are the results of one run of the simulation to determine a reasonable number of required sections to obtain a positive result with $95 \%$ confidence. The regression equation and $\mathrm{R} 2$ value listed is computed with a simple linear regression

\section{Discussion}

Breast implant anaplastic large cell lymphoma is an uncommon disease that may be missed by clinicians, radiologists, surgeons and pathologists because of the variable and fragmented clinical, radiological and microscopic
Table 4 Example on how to use a regression model formula to calculate the number of sections that will hit a lesion (\% area of tumor involvement) with $95 \%$ certainty

\begin{tabular}{lllll}
\hline Case \# & $\begin{array}{l}\text { Observed } \\
\text { \% Area of tumor } \\
\text { involvement } \\
\text { coverage) }\end{array}$ & $\begin{array}{l}\text { Observed } \\
\text { \# Sections } \\
\text { pos/total } \\
\text { sections }\end{array}$ & $\begin{array}{l}\text { Observed } \\
\% \text { Sections } \\
\text { positive }\end{array}$ & $\begin{array}{l}\text { Calculated } \\
\text { \# Sections } \\
\text { needed for } \\
\text { tumor } \\
\text { detection }\end{array}$ \\
\hline 16 & 5 & $1 / 14$ & 7 & 25 \\
21 & 10 & $10 / 50$ & 20 & 14 \\
40 & 20 & $5 / 28$ & 18 & 9 \\
11 & 30 & $60 / 93$ & 64 & 7 \\
\hline
\end{tabular}

In a regression model, there are two constants $\mathrm{b} 0$ and $\mathrm{b} 1$ that minimize the error between a line and all the data points using this equation: $y=$ $\mathrm{b} 0 / x+\mathrm{b} 1$, where $\mathrm{b} 0=106.9, \mathrm{~b} 1=3.6 ; x$ is the $\%$ area of coverage (observed) To illustrate on how to use the formula, we have selected case 21 in Table 2 . \# sections $=106.9 / 10+3.6=10.7+3.6=14.3$ that can be rounded to 14 sections. Observed area of tumor coverage (second column) and positive sections compared with expected number of sections until first detection of anaplastic large cell lymphoma in capsule. Therefore, we can enunciate: if a tumor of that size $(10 \%$ of the capsule area for case 21$)$ is not hit after 14 hits, it is very unlikely that additional sections will detect a tumor of that size. Since we found that the median \% area of tumor coverage is $10 \%$ for 44 cases of breast implant anaplastic large cell lymphoma we chose case 21 for this reason, however, for completion, other cases with different $\%$ coverage are also displayed. Note that a lower number of sections is calculated to be needed as \% coverage increases

appearance of this lymphoma. Particularly for pathologists, in cases clinically suspicious for breast implant anaplastic large cell lymphoma, lymphoma may not be readily apparent at the time of gross examination; the neoplasm may resemble a necrotizing, infectious or inflammatory process with fibrinoid or granular material located on the inner aspect of the breast capsule. In those cases where the capsule wall is involved, there may be thickening of the wall or mural tan-white nodules, but these findings may be overlooked if they are inconspicuous. Thus, improper assessment and processing of capsule specimens with breast implant anaplastic large cell lymphoma could generate false negative results. For example, unintentional separation of the fibrinoid material with tumor cells from the capsule or incorrect orientation of the sections ultimately can hamper the ability of the pathologist to identify scarce lymphoma cells microscopically and consequently delaying diagnosis. Inadequate sampling also may explain some cases in which tumor cells were identified in cytology specimens, but not in tissue samples. The value provided from appropriate staging lies in that depth of invasion (higher stage) has a worst prognosis when compared with tumor cells confined to the luminal side of the capsule [4]. Furthermore, the assessment of margins derived from inking and orienting the specimens as we propose enables the assessment of resection margins, additional essential information to report 
in breast implant anaplastic large cell lymphoma, since complete excision is the cornerstone for cure of this lymphoma [15].

In this study, we confirm the presumption that lymphoma can be identified in capsulectomies without a grossly identifiable tumor mass. The extent of the tumor cells over the luminal side of the capsule is variable, and ranges from 0 to $90 \%$ with a median of $10 \%$. With current practices, however, it was not possible to determine whether the tumor was a continuum or multifocal, nor was it possible to determine a preferential location of affected areas. One might consider the possibility of "floating" lymphoma cells falling down to the inferior aspect of the capsule just by simple gravity, but this hypothesis is mere speculation and could not be confirmed in this study. Of utmost interest, although most cases showed lymphoma confined to the luminal side of the capsule, $36 \%$ of cases had deep invasion into the capsule or beyond the capsule in the form of small nodular thickening of capsules, barely or not perceptible on gross examination that microscopically revealed lymphoma cell aggregates. The immediate implication of these data is that undetected tumor may explain recurrence or progression of disease if margins are not adequately identified and sampled. These limitations can be overcome with a systematic evaluation of complete capsulectomy specimens as we have proposed.

On the basis of the results in this study and a better understanding of breast implant anaplastic large cell lymphoma in general, we suggest that the College of American Pathologists recommendations on the workup of breast implants be updated. In this study, there was wide variability in the number of sections and paraffin blocks submitted per case. For example, in one case in which the sections of capsule were small $(1 \mathrm{~cm})$, there were 147 blocks with adequate orientation of capsule. The overall inner surface for evaluation in this case was greater than another case in which the sections were 2 or $3 \mathrm{~cm}$ long, but there were less than five blocks submitted. Moreover, as mentioned above, poor orientation of the capsule also influences the detection of tumor. In the cases grossed using our protocol, most capsule sections submitted were no less than $2 \mathrm{~cm}$ in length. We contend that two is a reasonable number of $2 \mathrm{~cm}$ in length tissue sections to fit into one cassette for obtaining adequate quality. Discretion and future experience may dictate if more samples can safely fit in one cassette while maintaining quality sections.

As an appendix, we propose a protocol for handling and processing breast capsules without a grossly identifiable mass in patients with suspicion of breast implant anaplastic large cell lymphoma. Based on our experience we present a theoretical rationale for sampling specimens without a distinctive tumor lesion using a mathematical model that includes the extent of tumor cells and number of positive sections, allowing a calculation of the minimum number of sections to identify $95 \%$ of cases of lymphoma. We highlight the value of preoperative evaluation, surgical evaluation of preferred en bloc resection of capsulectomy and implant with orientation of the excised specimen, accrual of fresh specimens for specialty testing such as cytopathology and flow cytometry immunophenotypic analysis, overnight fixation and systematic sampling of the capsule with the minimum number of sections to identify at least $95 \%$ of positive specimens. We also emphasize inking margins and on-edge embedding and sectioning given that about one third of cases had infiltration by lymphoma within or through the capsule, as well as the previously described risk of events and lymph node involvement. We suggest this protocol facilitates optimal handling, sampling and reporting of complete capsulectomies from patients with breast implant anaplastic large cell lymphoma without a grossly identifiable tumor mass.

By mapping the capsule in a systematic way after surgical orientation, grossly not identifiable anaplastic large cell lymphoma can be localized in the specimen and additional adjacent sections may be submitted for microscopic evaluation. We believe that proper orientation of the capsule is important to increase the rate of detection of breast implant anaplastic large cell lymphoma and to determine the distribution and extension of the disease into the capsule wall and pericapsular soft tissues. We believe that adherence to this protocol may lead to reproducible findings and mapping of lymphoma cells in involved capsules, as shown in Box 1. Furthermore, we recently showed that pathologic staging has prognostic significance and therefore excellent quality of orientation will facilitate better assessment for staging [18]. Proper orientation and histologic sectioning "on edge" allowed us to appreciate the entire capsule wall thickness, from the luminal surface to the outer soft tissues and margins. Additionally, our approach is necessary to determine margin status since the presence of a positive margin indicates the need for re-excision or other treatment modalities, such as chemotherapy or radiation therapy, although currently there is no consensus approach $[4,6,14,19]$. Importantly, breast implant anaplastic large cell lymphoma should be considered a localized disease that can be cured by complete resection, unlike systemic anaplastic large cell lymphoma [4].

It is important to emphasize that the gross examination approach we propose is recommended only for those patients in whom there is a suspicion for breast implant anaplastic large cell lymphoma and not for all routinely grossed breast capsules. In cases where the suspicion level for breast implant anaplastic large cell lymphoma is very low, we propose a protocol for gross examination of uninvolved breast implant capsules (Box 2). Due to the uncommon frequency of the disease, even referring 
surgeons at community institutions may not be aware of this entity and consequently may not alert the pathologists of the relevant clinical history. Education and sharing of information from all specialists (oncologists, radiologists, surgeons, and pathologists) are crucial to performing state-ofthe-art care for these patients. Importantly, institutions like the Food and Drug Administration have recognized the importance of breast implant anaplastic large cell lymphoma. Through their MedWatch program, The Food and Drug Administration Safety Information and Adverse Event Reporting Program, the Food and Drug Administration has publicized details of the disease and provided a website and specifically recommends reporting new cases of breast implant anaplastic large cell lymphoma to the PROFILE registry (www.thepsf.org/PROFILE) [18, 20, 21].

Following the recommended protocol, personnel and fellows are trained to follow the recommendations as outlined in Box 1-3. Before gross evaluation, we determine if the specimen resulted from partial or complete capsulectomy and if the capsule is intact (Fig. 4a) The assessment for obvious fluid collections around an implant is performed. Careful fluid aspiration with a sterile syringe and needle are done for subsequent cytologic and/or flow cytometry analyses (Fig. 4b). Then, we orient the specimen following the surgeons' indications and identify six landmark positions to guide subsequent sampling: superior, anterior, inferior, posterior, medial, and lateral surfaces. For cases without orientation and no distinct lesion, these positions are assigned arbitrarily. On en bloc specimens, we make a horizontal incision on the central portion of the posterior surface of the capsule (Fig. 4c), and carefully remove and save any residual fluid. Next, vertical incisions of the posterior surface are made for careful implant removal (Fig. 4d-f). Any clot or pieces of floating fibrin/ tissue contents that may be present within the capsule cavity are submitted for histologic and/or cytological examination including cell block as well as other ancillary studies (Cytolite, cellblock preparation). Gross evaluation of the implant is performed and we document the type of implant, the surface type, and if it was intact or previously ruptured. Then the implant and capsule are measured.

After, visual and palpation evaluation of the capsule for any distinct lesion or mass is performed; its position is recorded and described as granular, nodular, indurated, fibrinoid, hemorrhagic or fleshy. The inspection is completed and the capsule is pinned flat with the outer surface facing the paraffin board and submerged in $10 \%$ buffered formalin overnight to allow for fixation (Fig. 5). Once
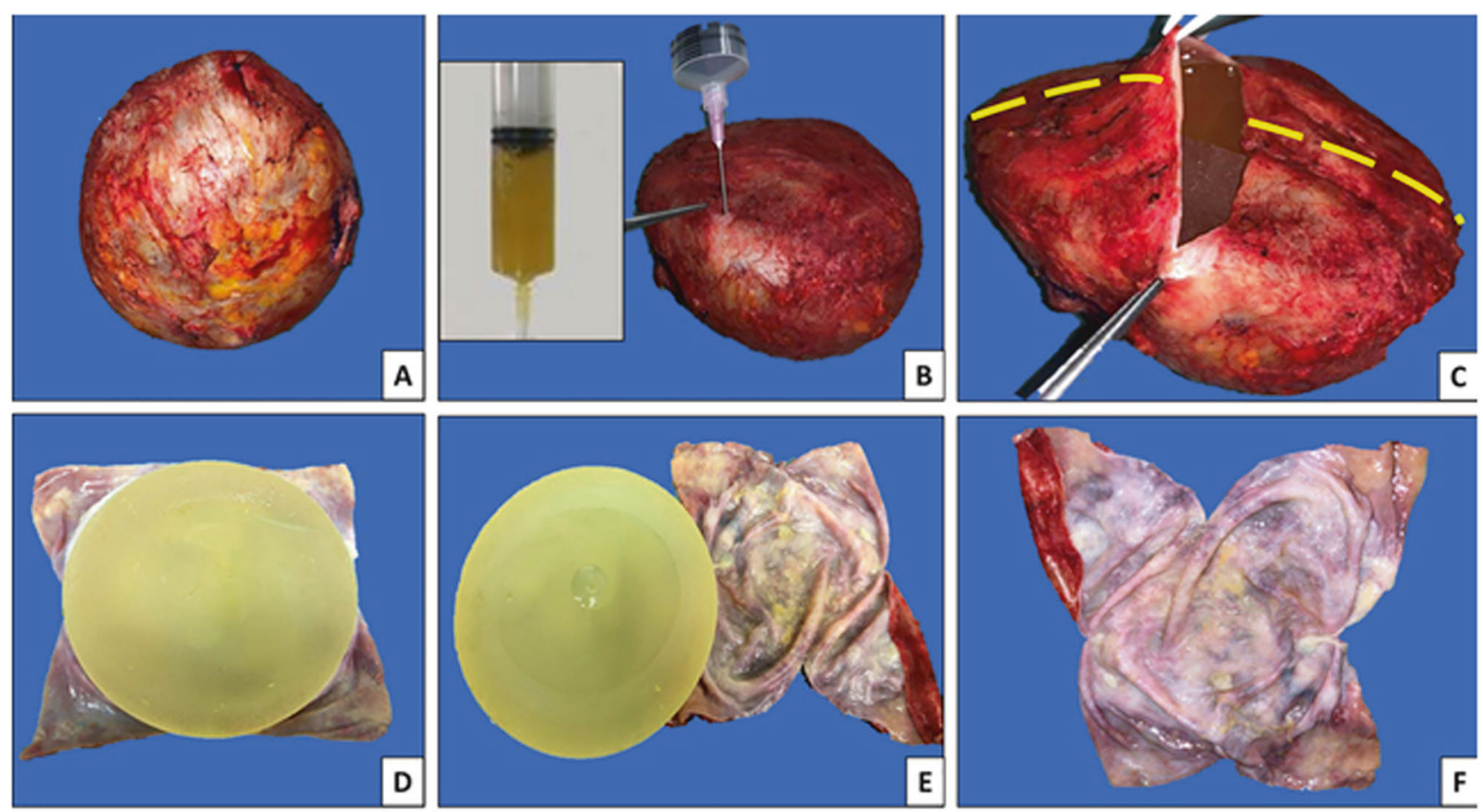

Fig. 4 Steps for proper handling and processing of capsules and implants with suspicion of breast implant anaplastic large cell lymphoma. a En bloc resection of capsule containing the implant. Note the specimen is oriented by the surgeon: Long stitches denote lateral margin; short stitches denote the superior margin. b Preoperatively the presence of peri-implant effusion was determined. A $50-\mathrm{mL}$ syringe allows aspiration of the fluid/effusion, as noted in the inset, to be sent for cytopathology and flow cytometry immunophenotype analyses. c Incision on the posterior surface, from lateral to medial allows visualization of remnants of fluid and implant. Additional vertical incisions (interrupted lines) allows for a better exposure of the luminal surface of the capsule. d Implant in situ with opened capsule displaying the luminal surface. e Detachment of implant. f Flat-opened capsule displaying the luminal surface 
properly fixed, the outer aspect of the capsule is inked as follows: anterior surface in yellow, posterior in black, lateral in orange, medial in red, superior in blue, and inferior in green (Fig. 6a). The ink on the outer surface of the capsule is also actually the surgical margin. Then, we sample any distinct lesions and record the location. Any mass or thickening of the capsule should be sampled generously. If a distinct lesion is not identified, two representative sections of each of six aspects of the capsule should be taken (Fig. 6b). Two slivers of approximately $2 \mathrm{~cm}$ in length usually fit into one cassette (Fig. 7). This procedure allows maintaining the capsule as one specimen as much as possible for the possibility further sampling is required. Decalcification of the capsule sections may be needed if they are too hard and calcified. We take photographs of the presampled specimen, contents of the capsule after the first incision, the implant surface and inscription, the luminal

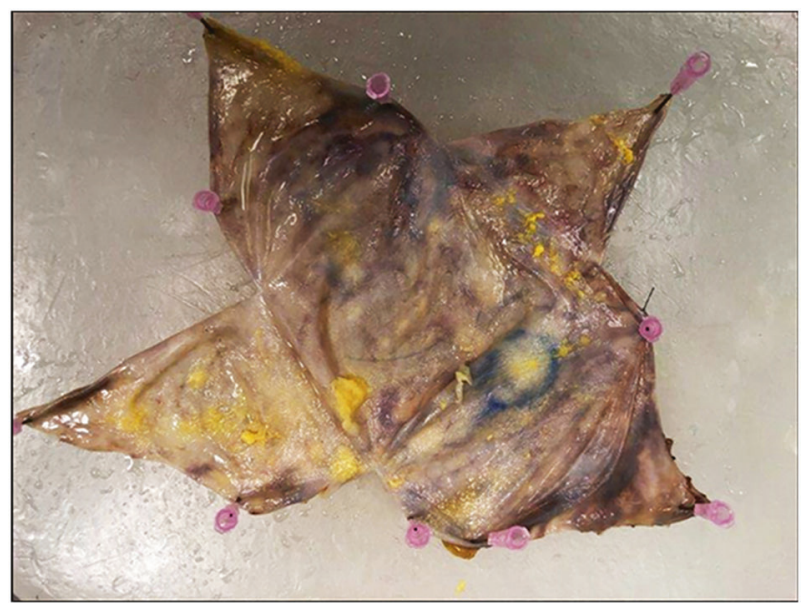

Fig. 5 Flat-opened capsule displaying the luminal surface pinned to a paraffin board, ready to be submerged in $10 \%$ buffered formalin for appropriate fixation. No obvious masses or lesions are seen grossly side of the capsule, fresh and post-fixation, and cross sections of suspected nodular or thickened areas. Histologic sections on edge allow evaluation of the luminal and abluminal surfaces of the capsule.

We propose a gross procedure for the handling of pathologic specimens suspected to have breast implant anaplastic large cell lymphoma. Our approach includes a pre-operative as well as a post-operative evaluation of the case (see Box 1), orientation of the specimen for thorough sampling and look-back for any lesion identified on subsequent examination. The approach we propose is a standard procedure at our institution and every suspected case of breast implant anaplastic large cell lymphoma includes previous fixation, pinning flat of the capsule, mapping the specimen with respective sampling, and proper orientation of the capsule for sectioning (on edge) (see Box 2). This method renders excellent results for detection of breast implant anaplastic large cell lymphoma cells and to a certain degree, for evaluation of the disease extent. All the cases that have been processed using this procedure have been diagnosed as breast implant anaplastic large cell lymphoma or at least showed atypical cells, which prompted the use of immunohistochemistry to confirm the diagnosis. An additional advantage of the proper orientation of the capsule tissue sections, is the identification of histopathological findings that have not been previously addressed in other studies [22]. Although one may argue that once the diagnosis of breast implant anaplastic large cell lymphoma is made in cytology, the identification of tumor in the capsule does not have a significant clinical relevance, we believe it is justified to determine the pathologic staging and status of margins, as mentioned above. Moreover, there are instances where a cytology sample is not obtained, or the cytology material is not definitive for diagnosis or is suspicious for anaplastic large cell lymphoma; the evaluation of extremely necrotic specimens is additionally challenging,

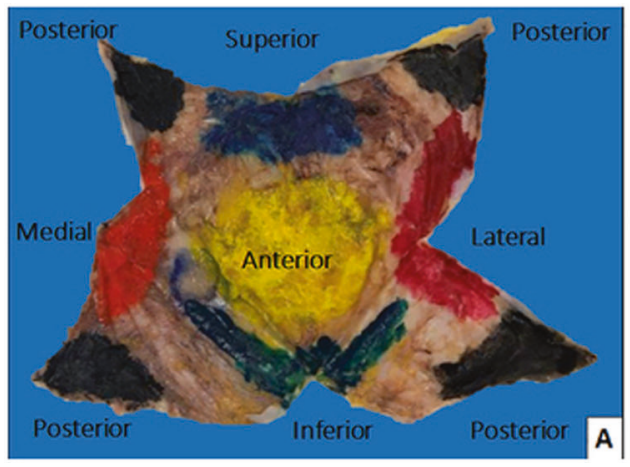

Fig. 6 a After overnight fixation, the outer aspect of the capsule is inked as follows: anterior surface in yellow, superior in blue, inferior in green, medial in red, lateral in orange, and posterior in black. b Inked specimen is sampled and is displayed after two representative

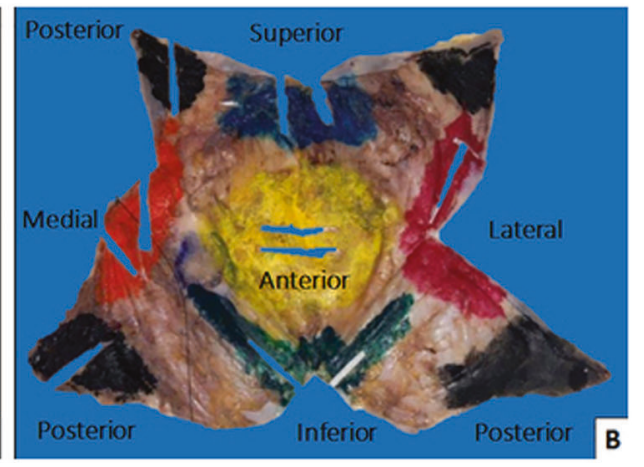

sections of each of six aspects of the capsule were taken and appear as empty rectangles. We strongly recommend taking the samples of capsule facing the luminal side 


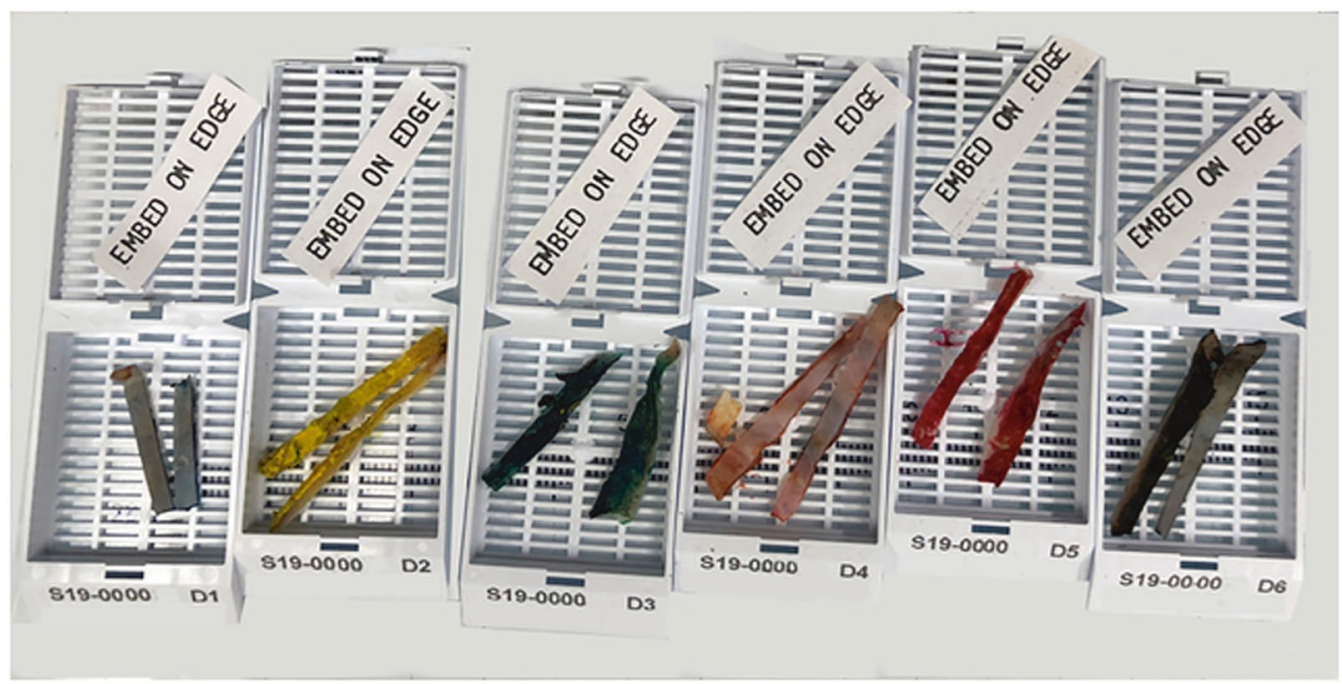

Fig. 7 Two sections of approximately $2-3 \mathrm{~cm}$ in length each are submitted per each of the aspects of the capsule. It is important to indicate to the histotechnologist to "embed on edge" the sections, so as to visualize the luminal surface of the capsule in its entirety

and evaluation of the capsule may be the only available material for diagnosis.

In summary, in this study we assessed the extent of lymphoma cells and numbers of positive sections in complete capsulectomies from patients with breast implant anaplastic large cell lymphoma without a grossly identifiable tumor mass. We present our suggestions for best practices for the optimal handling, sampling, and reporting of these specimens and we present a mathematic rationale for sampling when a lesion is not visible and randomly distributed.

Acknowledgements We thank all personnel in the Departments of Hematopathology and Pathology who supported us to perform all that was required for this project.

\section{Compliance with ethical standards}

Conflict of interest RNM received compensation from Allergan Inc. for advisory expert opinion in a one-day meeting. The remaining authors declare that they have no conflict of interest.

Publisher's note: Springer Nature remains neutral with regard to jurisdictional claims in published maps and institutional affiliations.

\section{References}

1. Feldman AL, Harris NL, Stein H, Campo E, Kinney MC, Jaffe ES, et al. Breast implant-associated anaplastic large cell lymphoma, In: Swerdlow SH, Campo E, Harris NL, Jaffe ES, Pileri SA, Stein H, Thiele J, editors. WHO Classification of Tumours of Haematopoietic and Lymphoid Tissues (Revised 4th edition). Lyon: IARC; 2017. p 421-2.

2. de Jong D, Vasmel WL, de Boer JP, Verhave G, Barbe E, Casparie MK, et al. Anaplastic large-cell lymphoma in women with breast implants. JAMA. 2008;300:2030-5.
3. Roden AC, Macon WR, Keeney GL, Myers JL, Feldman AL, Dogan A. Seroma-associated primary anaplastic large-cell lymphoma adjacent to breast implants: an indolent T-cell lymphoproliferative disorder. Mod Pathol. 2008;21:455-63.

4. Miranda RN, Aladily TN, Prince HM, Kanagal-Shamanna R, de Jong D, Fayad LE, et al. Breast implant-associated anaplastic large-cell lymphoma: long-term follow-up of 60 patients. J Clin Oncol. 2014;32:114-20.

5. Keech JA Jr., Creech BJ. Anaplastic T-cell lymphoma in proximity to a saline-filled breast implant. Plast Reconstr Surg. 1997;100:554-5.

6. Kim B, Predmore ZS, Mattke S, van Busum K, Gidengil CA. Breast implant-associated anaplastic large cell lymphoma: updated results from a structured expert consultation process. Plast Reconstr Surg Glob open. 2015;3:e296.

7. Kricheldorff J, Fallenberg EM, Solbach C, Gerber-Schafer C, Rancso C, Fritschen UV. Breast Implant-Associated Lymphoma. Dtsch Arzteblatt Int. 2018;115:628-35.

8. Aladily $\mathrm{TN}$, Medeiros LJ, Amin MB, Haideri N, Ye D, Azevedo SJ, et al. Anaplastic large cell lymphoma associated with breast implants: a report of 13 cases. Am J Surg Pathol. 2012;36:1000-8.

9. Quesada AE, Medeiros LJ, Clemens MW, Ferrufino-Schmidt MC, Pina-Oviedo S, Miranda RN. Breast implant-associated anaplastic large cell lymphoma: a review. Mod Pathol. 2019;32:166-88.

10. Adrada BE, Miranda RN, Rauch GM, Arribas E, KanagalShamanna R, Clemens MW, et al. Breast implant-associated anaplastic large cell lymphoma: sensitivity, specificity, and findings of imaging studies in 44 patients. Breast Cancer Res Treat. 2014;147:1-14.

11. Anonym. Revised CAP guidelines for prosthetic breast implants. In: CAP Today. Vol. 9, Chicago: College of American Pathologists; 1995.

12. Zarbo RJ, Nakhleh RE. Surgical pathology specimens for gross examination only and exempt from submission: a College of American Pathologists Q-Probes study of current policies in 413 institutions. Arch Pathol Lab Med. 1999;123:133-9.

13. Lester SC. Manual of Surgical Pathology: Expert Consult. Elsevier Health Sciences. London: Elsevier Health Sciences; 2010.

14. Brody GS, Deapen D, Taylor CR, Pinter-Brown L, House-Lightner SR, Andersen JS, et al. Anaplastic large cell lymphoma occurring in 
women with breast implants: analysis of 173 cases. Plast Reconstr Surg. 2015;135:695-705.

15. Clemens MW, Medeiros LJ, Butler CE, Hunt KK, Fanale MA, Horwitz $\mathrm{S}$, et al. Complete surgical excision is essential for the management of patients with breast implant-associated anaplastic large-cell lymphoma. J Clin Oncol. 2016;34:160-8.

16. Dielman TE. Applied Regression Analysis: A Second Course in Business and Economic Statistics. 4, illustrated ed. Boston, MA: Brooks/Cole Thomson Learning; 2005.

17. Giardini R, Piccolo C, Rilke F. Primary non-Hodgkin's lymphomas of the female breast. Cancer. 1992;69:725-35.

18. Administration UFaD. Anaplastic large cell lymphoma (ALCL) in women with breast implants: preliminary FDA findings and analyses. 2011. http://www.fda.gov/MedicalDevices/Productsa ndMedicalProcedures/ImplantsandProsthetics/BreastImplants/ ucm239995.htm. Accessed 30 Nov 2014.
19. Gidengil CA, Predmore Z, Mattke S, van Busum K, Kim B. Breast implant-associated anaplastic large cell lymphoma: a systematic review. Plast Reconstr Surg. 2015;135:713-20.

20. Administration UFaD. FDA medical device communication: Reports of anaplastic large cell lymphoma (ALCL) in women with breast implants. 2011. http://www.fda.gov/medicaldevices/safety/a lertsandnotices/ucm240000.htm. 2011. Accessed 5 Dec 2014.

21. McCarthy CM, Loyo-Berrios N, Qureshi AA, Mullen E, Gordillo $\mathrm{G}$, Pusic AL, et al. Patient registry and outcomes for breast implants and anaplastic large cell lymphoma etiology and epidemiology (PROFILE): initial report of findings, 2012-2018. Plast Reconstr Surg. 2019;143:65S-73S.

22. Taylor CR, Siddiqi IN, Brody GS. Anaplastic large cell lymphoma occurring in association with breast implants: review of pathologic and immunohistochemical features in 103 cases. Appl Immunohistochem Mol Morphol. 2013;21:13-20.

\section{Affiliations}

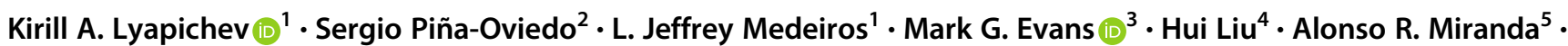
Kelly K. Hunt ${ }^{6} \cdot$ Mark W. Clemens $^{7}$ - John M. Stewart ${ }^{8} \cdot$ Mitual B. Amin $^{9}$ - Andres E. Quesada ${ }^{10}$. Siaw Ming Chai ${ }^{11}$. Arianna Di Napoli ${ }^{12}$ - Arthy Yoga ${ }^{13} \cdot$ Sanat K. Dave ${ }^{1}$ Ignacio I. Wistuba ${ }^{14} \cdot$ Yun Wu $^{8} \cdot$ Carlos E. Bueso-Ramos $^{1}$. Ellen J. Schlette ${ }^{1} \cdot$ Maria C. Ferrufino-Schmidt ${ }^{14} \cdot$ Sanam Loghavi $\mathbb{D}^{1} \cdot$ Joseph D. Khoury $^{1}{ }^{1} \cdot$ Ken H. Young $^{1}$. Roberto N. Miranda $\mathbb{1}^{1}$

1 Department of Hematopathology, The University of Texas MD Anderson Cancer Center, Houston, TX, USA

2 Department of Pathology, University of Arkansas for Medical Sciences, Little Rock, AR, USA

3 Department of Pathology and Laboratory Medicine, University of California, Irvine, Orange, CA, USA

4 Department of Pathology, Xuzhou Medical Universtiy, Xuzhou, Jiangsu Province, PR China

5 Department of Mathematics, University of Houston, Houston, TX, USA

6 Department of Breast Surgical Oncology, The University of Texas MD Anderson Cancer Center, Houston, TX, USA

7 Department of Plastic Surgery, The University of Texas MD Anderson Cancer Center, Houston, TX, USA

8 Department of Pathology, The University of Texas MD Anderson Cancer Center, Houston, TX, USA
9 Department of Pathology and Laboratory Medicine, Oakland University William Beaumont School of Medicine, Royal Oak, MI, USA

10 Department of Pathology, Memorial Sloan Kettering Cancer Center, New York City, NY, USA

11 Department of Anatomical Pathology, PathWest Laboratory Medicine, QEII Medical Centre, Perth, WA 6009, Australia

12 Pathology Unit, Department of Clinical and Molecular Medicine, Sant'Andrea Hospital, School of Medicine and Psychology, Sapienza University of Rome, Rome, Italy

13 Breast Surgical Oncologist, Texas Oncology, Houston, TX, USA

14 Department of Translational Molecular Pathology, The University of Texas MD Anderson Cancer Center, Houston, TX, USA 